\title{
IMPROVED INDOOR POSITIONING USING BLE DIFFERENTIAL DISTANCE CORRECTION AND PEDESTRIAN DEAD RECKONING
}

\author{
Y. T. Tang ${ }^{1}$, Y. T. Kuo ${ }^{1}$, J. K. Liao ${ }^{1}$, K. W. Chiang ${ }^{1, *}$ \\ ${ }^{1}$ Department of Geomatics, National Cheng Kung University, No. 1, University Road, Tainan, Taiwan - \\ (sugar.14387, yuki31210, cacalut1690, kwchiang72)@gmail.com
}

KEY WORDS: indoor positioning, Bluetooth low energy (BLE), received signal strength indication (RSSI), differential distance correction (DDC), trilateration, pedestrian dead reckoning (PDR)

\begin{abstract}
:
Recently, indoor positioning becomes a popular issue because of its corresponding location-aware applications. Owing to the limits of the sheltered signal of satellites in indoor environments, one of the alternative scheme is Bluetooth Low Energy (BLE) technology. BLE device broadcasts Received Signal Strength Indicator (RSSI) for distance estimation and further positioning. However, in the complex indoor environment, the reflection, fading, and multipath effect of BLE make the variable RSSI and may lead to poor quality of RSSI. In this study, the concept called Differential Distance Correction (DDC) is similar to the Differential Global Navigation Satellite System (DGNSS). This method can eliminate some residuals and further improve the results with the corrected distance. On the other hand, Pedestrian Dead Reckoning (PDR) is another common indoor positioning method. PDR can propagate the next position from the current position by the implemented of inertial sensors. Despite that, the error of inertial sensors would accumulate with time and walking distance, which position update is required for restraining the drift. Accordingly, the two indoor positioning methods have their strong and weak point. BLE-based positioning is absolute positioning, while PDR is relative positioning. This study proposes a concept that combines the two methods. The pedestrian receives the RSSI and records the information from inertial sensors simultaneously. Through the complementary of two methods, the positioning results would be improved from $29 \%$ to $66 \%$ according to different travelled distance.
\end{abstract}

\section{INTRODUCTION}

With new technological advances, people are more and more dependent on the conventional technology in the positioning system. Nowadays, the Global Navigation Satellite System (GNSS) is already a part of daily life, while the signals are blocked in the indoor environment. Additionally, indoor positioning can apply various application i.e. product tracking, indoor navigation or smart city. Although people spend about $90 \%$ of their time indoors (Velux, 2018), applications indoors still have difficultly to achieve the same level of positioning accuracy, continuity and reliability as outdoors (Basiri et al., 2017). Hence, the indoor positioning has gained popularity in recent years. Many researchers are devoted to developing various indoor positioning approaches.

A kind of technique in indoor positioning is using wireless signals to replace GNSS signals, such as Wi-fi, Infrared (IR), Radio Frequency Identification (RFID), and Ultra-wideband (UWB). Among them, Bluetooth Low Energy (BLE), version 4.0 of Bluetooth, is the most feasible choice on account of cost, power consumption, deployment, distance of transmission, etc. BLE device, beacon, is the transmitter that can broadcast Received Signal Strength Indicator (RSSI). RSSI can be converted to distance, and the distance is the main material in the positioning approach. That is, the RSSI is vital to the result of positioning. With RSSI, three kinds of approaches can be adopted: trilateration, fingerprinting, and proximity detection (Čabarkapa et al, 2015). The main challenge to BLE-based indoor positioning is to reduce the effect of the environment changes including reflection, fading, and multipath effect (Zhuang et al, 2016). Hence, the concept called differential distance correction (DDC) is introduced to eliminate these effects. The fingerprints should be trained previously, which is not suitable for applying DDC. As for proximity detection, the algorithm selects the location information of beacon broadcasting the highest RSSI, which is also not applicable for DDC. Consequently, the DDC strategy is based on trilateration method, which is similar to the Differential Global Navigation Satellite System (DGNSS).

Besides the wireless signals, another technique to positioning in the indoor environment is Pedestrian Dead Reckoning (PDR). PDR is an approach based on the low-cost sensors embedded in the smartphone. It is independent of the environmental factor and there is no need to deploy transmitters in the field. The error accumulated with time and walking distance is the critical defect of PDR.

The two indoor positioning methods have their strong and weak point. BLE-based positioning is an absolute positioning, which obtains higher positions in the long term and is time-independent. However, the variation of the signal is a significant defect. As for PDR, it is a relative positioning, of which error accumulates over time, but it's self-contained because of no signal interfered. Hence, this paper proposes a method to combine the two indoor positioning approach. Using the results of BLE updates the position calculated by PDR.

\section{METHODOLOGY}

The overall process of this experiment is shown in Figure 1. The starting point is known in advance. In the beginning, the PDR is used to locate the person's position. When the pedestrian stops for a while, the method changes and the results of BLE with DDC replaces the former location calculated by PDR at that time. While the pedestrian keeps going, with the updated coordinate as a new initial position, the error caused by time in PDR would be reduced. The following sections will explain more in each step.

\footnotetext{
* Corresponding author
} 


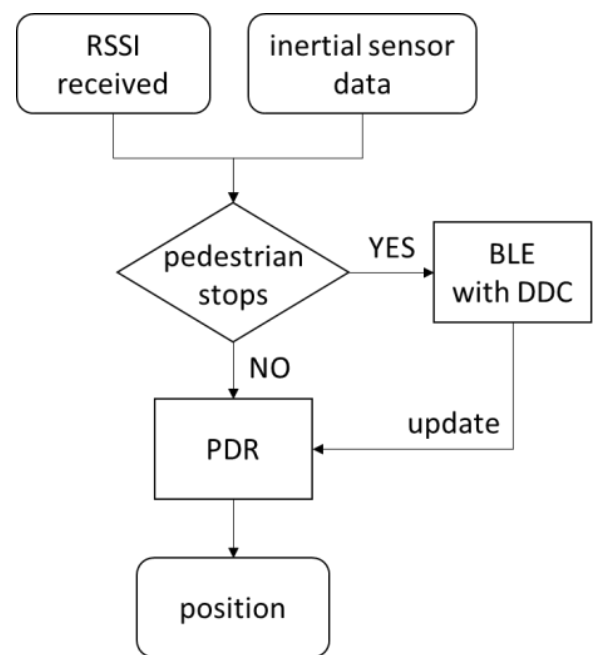

Figure 1. The flow chart of experiment

\subsection{Trilateration}

Trilateration is a classical positioning technique that utilizes the estimated distances to determine the location of the target. Each distance between transmitter and receiver acts as the radius of a circle, and the intersection of circles is the location of the receiver, which is represented in Figure 2.

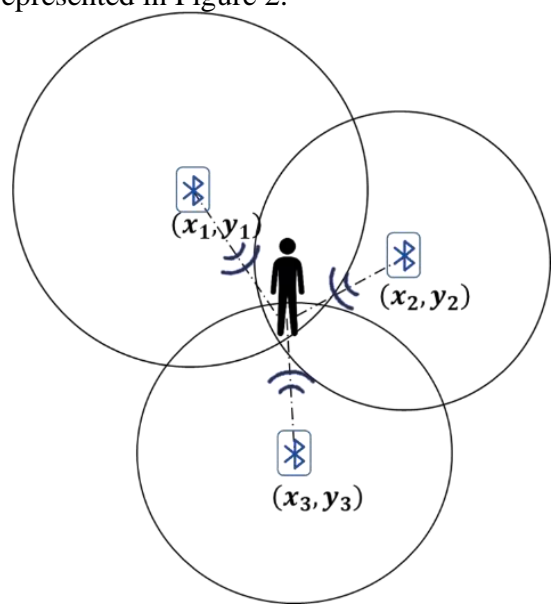

Figure 2. The diagram of trilateration

Since the estimated distance doesn't exactly correct, the intersection is an area instead of a point. As a result, the least squares method is adopted to determine the optimal solution. The equation is described in the following equation (Murphy, 2007):

$$
\delta \vec{D}=D_{i}-\widehat{D}_{i}=H \delta \vec{r}
$$

The parameter above can be represented by Equation (2) to (5):

$$
\begin{aligned}
& D_{i}=\sqrt{\left(x-x_{i}\right)^{2}+\left(y-y_{i}\right)^{2}} \\
& \widehat{D}_{i}=\sqrt{\left(\hat{x}-x_{i}\right)^{2}+\left(\hat{y}-y_{i}\right)^{2}} \\
& H=\left[\begin{array}{cc}
\frac{\hat{x}-x_{1}}{\widehat{D}_{1}} & \frac{\hat{y}-y_{1}}{\widehat{D}_{1}} \\
\frac{\hat{x}-x_{2}}{\widehat{D}_{2}} & \frac{\hat{y}-y_{2}}{\widehat{D}_{2}} \\
\vdots & \vdots \\
\frac{\hat{x}-x_{n}}{\widehat{D}_{n}} & \frac{\hat{y}-y_{n}}{\widehat{D}_{n}}
\end{array}\right]
\end{aligned}
$$

$$
\delta \vec{r}=\left[\begin{array}{l}
x-\hat{x} \\
y-\hat{y}
\end{array}\right]
$$

where

$D_{i}=$ real distance between the ith beacon and the target

$\widehat{D}_{i}=$ estimated distance between the ith beacon and the target

$\delta \vec{D}=$ error of the distance

$H=$ design matrix

$\delta \vec{r}=$ error between the real and estimated coordinate of the target

$x_{i}, y_{i}=$ coordinates of ith beacon

$x, y=$ real coordinates of the target

$\hat{x}, \hat{y}=$ estimated coordinates of the target

Next, the least squares method shown in Equation (6) is utilized to minimize the sum of the squares of the errors.

$$
\delta \vec{r}=\left(H^{T} H\right)^{-1} H^{T} \delta \vec{D}=\left[\begin{array}{l}
x-\hat{x} \\
y-\hat{y}
\end{array}\right]=\left[\begin{array}{l}
\delta x \\
\delta y
\end{array}\right]
$$

Finally, Equation (7) shows the optimal solution can be obtained by iteration.

$$
\left[\begin{array}{l}
\hat{x} \\
\hat{y}
\end{array}\right]_{k+1}=\left[\begin{array}{l}
\hat{x} \\
\hat{y}
\end{array}\right]_{k}+\left[\begin{array}{l}
\delta x \\
\delta y
\end{array}\right]_{k}
$$

\subsection{Differential Distance Correction}

The distance converted from RSSI is an estimated value owing to the influenced by the environment. Consequently, the method called differential distance correction (Kuo et al., 2017), which is similar to DGNSS is proposed. The basic assumption of that approach is that the two stations not far from each other are supposed to be affected by the same effect in the environment. Given A known station, reference station in the field, there are two distances between the beacon and the reference station. One is the real distance calculated by Euclidean distance formula and the other is the estimated distance converted from RSSI. The difference between two distances can be regarded as the residual in other near unknown station because of the assumption mentioned above, as Figure 3 shown.

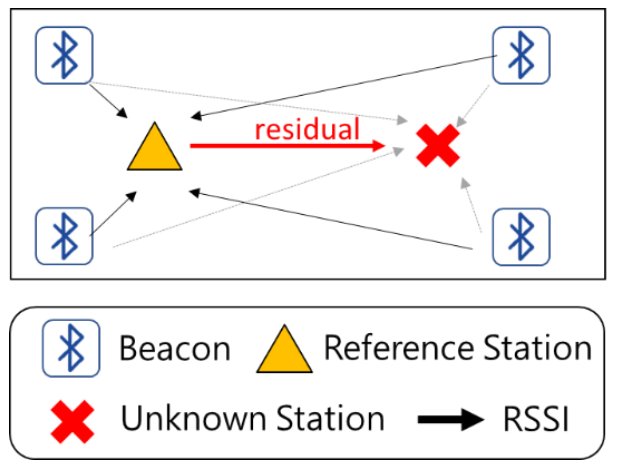

Figure 3. The diagram of differential distance correction

In order to obtain the residual for every location in the field, Inverse Distance Weighted (IDW) is adopted. The residual at the unknown location can be derived from the equation as follow:

$$
r_{e s t}=\sum_{j=1}^{N} w_{j} \times r_{j}
$$

where $r_{j}=$ estimated residual of the $\mathrm{j}^{\mathrm{th}}$ reference station $w_{j}=$ weight of the $\mathrm{j}^{\text {th }}$ reference station 
$w_{j}$ can be defined as Equation (9):

$$
w_{j}=\frac{d_{\bar{j}}^{u}}{\sum_{j=1}^{N} d_{\bar{j}}^{u}}
$$

where $\quad u=$ exponent parameter

$d_{\bar{j}}=$ distance between the $\mathrm{j}^{\text {th }}$ reference station and unknown location

$d_{\bar{\jmath}}$ is derived from Equation (10):

$$
d_{\bar{J}}=\sqrt{\left(X-x_{j}\right)^{2}+\left(Y-y_{j}\right)^{2}}
$$

where

$$
\begin{aligned}
& X, Y=\text { coordinates of the unknown location } \\
& x_{j}, y_{j}=\text { coordinates of the } \mathrm{j}^{\text {th }} \text { reference station }
\end{aligned}
$$

For each beacon, there is one residual map for one epoch. In the experiment, the 3-minutes data is received and is divided into 30 segmentation. Namely, there are 30 residual maps for each beacon. Figure 4 is an example of the residual map with four reference stations (The grid size is $0.5 \mathrm{~m} \times 0.5 \mathrm{~m}$ ). The initial position calculated by trilateration using original distance will be used to select the corresponding residual in the grid. With the residual, the corrected distance is obtained, and the trilateration will be utilized again to solve the new positioning result.

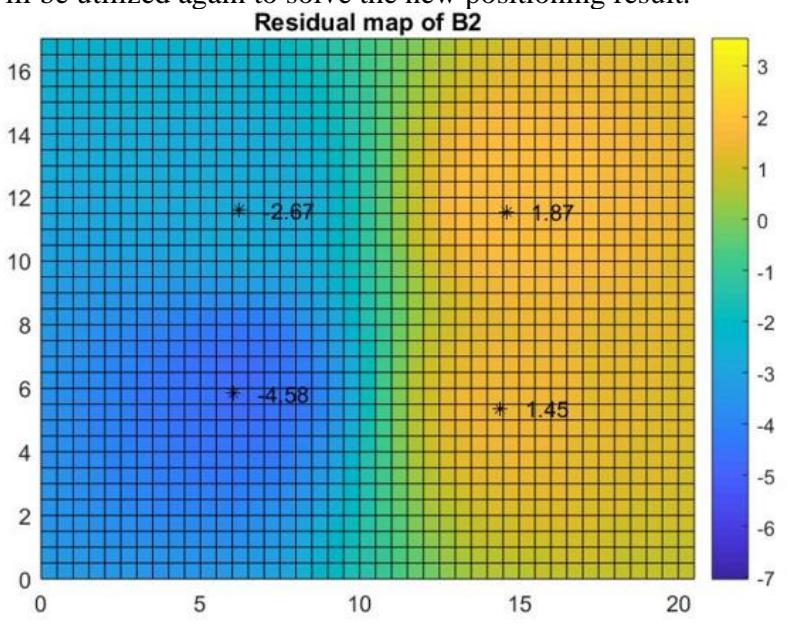

Figure 4. The residual map of beacon 2 at the last epoch

\subsection{Pedestrian Dead Reckoning (PDR)}

PDR is an approach based on an initial known position. Through the inertial sensor in the smartphone such as the accelerometer, magnetic compass, and gyroscope, the next position can be propagated (Chen et al, 2011) with Equation (11). As Figure 5 shows, with the position at $\mathrm{k}$ epoch, azimuth, and step length, the position at epoch $\mathrm{k}+1$ can be derived.

$$
\begin{aligned}
& N_{k+1}=N_{k}+S L_{k} \times \cos \varphi_{k} \\
& E_{k+1}=E_{k}+S L_{k} \times \sin \varphi_{k}
\end{aligned}
$$

where

$N_{k}, E_{k}=$ North and East coordinates at epoch k

$S L_{k}=$ step length at epoch $\mathrm{k}$

$\varphi_{k}=$ azimuth at epoch $\mathrm{k}$

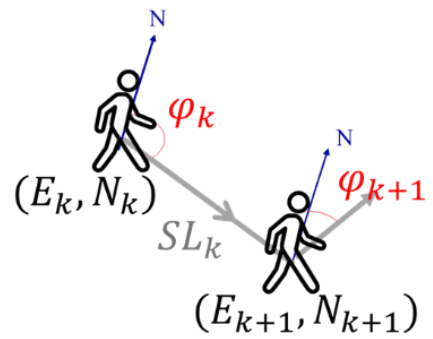

Figure 5. The concept of the PDR

In order to determine the next position, step detection, step length estimation, and azimuth recognition are necessary. Firstly, Step detection is needed to understand whether pedestrian walks or not. According to the variety of acceleration values, every step can be recognized by a given threshold. As Equation (12) shows, the acceleration of three dimensions should be considered.

$$
a_{\text {total }}=\sqrt{a_{x}^{2}+a_{y}^{2}+a_{z}^{2}}
$$

where

$$
a_{x}, a_{y}, a_{y}=\text { acceleration in } \mathrm{x}, \mathrm{y} \text {, and } \mathrm{z} \text { axis }
$$$$
a_{\text {total }}=\text { composition of acceleration }
$$

Secondly, Step lengths can be calculated by an empirical model (Chen et al, 2011), which is described in the following equation:

$$
S L=\left(0.7+a \cdot(H-1.75)+b \cdot \frac{(S F-1.79) \cdot H}{1.75}\right) \cdot
$$

where

$$
\begin{aligned}
& \text { SL = step length } \\
& \text { SF = step frequency } \\
& H=\text { height of the pedestrian } \\
& \mathrm{a}, \mathrm{b}=\text { two known parameters of the model } \\
& c=\text { personal factor that can be trained on-line }
\end{aligned}
$$

Lastly, the magnetic compass provides the absolute azimuth, which can be used directly, while gyroscope is a relative angular velocity, which should further derive the angle by Equation (14). Moreover, the relative angle from the gyroscope isn't useful without an initial angle prepared by the magnetic compass, which is given by Equation (15). The final azimuth form gyroscope is described in Equation (16).

$$
\begin{aligned}
& \text { Gyro }_{k+1}=-\omega_{k+1} \times\left(t_{k+1}-t_{k}\right) \cdot \frac{180^{\circ}}{\pi} \\
& \text { Gyro_A } A_{1}=\text { Gyro }_{1}+M a g_{1} \\
& \text { Gyro_A } A_{k+1}=\text { Gyro }_{k+1}+\text { Gyro_ } A_{k}
\end{aligned}
$$

where $\quad \omega_{k}=$ angular velocity from gyroscope at epoch $\mathrm{k}$ $t_{k}=$ time at epoch $\mathrm{k}$

Gyro $_{1}=$ angle derived from angular velocity at epoch 1

$M a g_{1}=$ azimuth from magnetic compass

at epoch 1

Gyroscope_ $A_{1}=$ initial azimuth

Gyroscope_ $A_{k}=$ azimuth at epoch $\mathrm{k}$

Gyroscope $_{\mathrm{k}}=$ angle derived from angular velocity at epoch $\mathrm{k}$

With step length and azimuth, the next position can be determined with Equation (11). 


\section{EXPERIMENT}

The experiment is carried out in the parking garage below the library of National Cheng Kung University (NCKU). The filed is an indoor environment with the length of $20 \mathrm{~m}$ and the width of $17 \mathrm{~m}$. The arrangement of the experimental field refers to Figure 6. There are nine beacons and four reference stations in the field. The pedestrian starts with the point named T12, walks clockwise, and stops for 3 minutes when backing to T12. Each of the participants would take 4 rounds for each experiment.

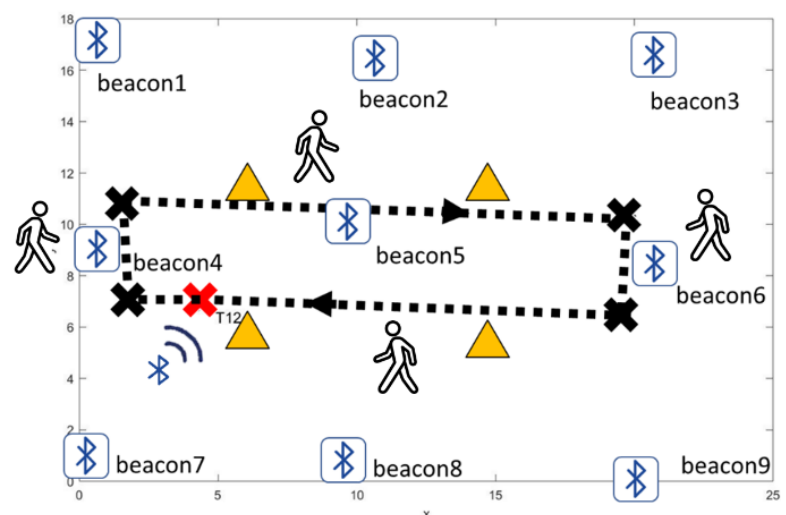

Figure 6. The illustration of the experiment field

\section{RESULTS AND DISCUSSION}

\subsection{BLE-based Indoor Positioning with DDC}

Figure 7 shows the positioning results of participant 1 stopping in T12. The timing of each is the end of circle1, 2, and 3. The blue points are the original results calculated by trilateration, and the green points are the results with the corrected distance. Table 1 summarizes the BLE positioning results respectively. With Differential Distance Correction, the error decreased noticeably.
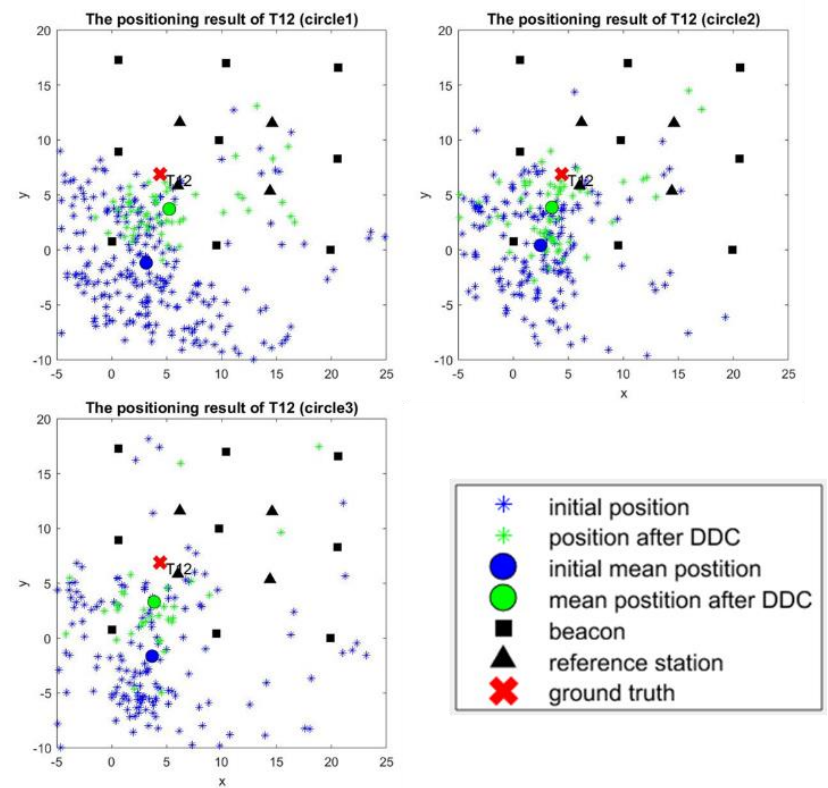

Figure 7. The BLE positioning results of participant 1

\begin{tabular}{|l|l|l|l|l|c|}
\hline \multicolumn{2}{|l|}{$\begin{array}{l}\text { positioning } \\
\text { results (m) }\end{array}$} & RMSE & STD & error & $\begin{array}{c}\text { improvement } \\
(\%)\end{array}$ \\
\hline \multirow{2}{*}{$\begin{array}{l}\text { End of } \\
\text { circle1 }\end{array}$} & original & 11.39 & 7.95 & 8.17 & \multirow{2}{*}{$59.98 \%$} \\
\cline { 2 - 5 } & DDC & 6.19 & 5.31 & 3.27 & \\
\hline \multirow{2}{*}{$\begin{array}{l}\text { End of } \\
\text { cicle2 }\end{array}$} & original & 9.85 & 7.18 & 6.76 & \multirow{2}{*}{$53.25 \%$} \\
\cline { 2 - 5 } & DDC & 6.44 & 5.66 & 3.16 & \\
\hline \multirow{2}{*}{$\begin{array}{l}\text { End of } \\
\text { circle3 }\end{array}$} & original & 12.28 & 8.82 & 8.57 & \multirow{2}{*}{$57.64 \%$} \\
\cline { 2 - 5 } & DDC & 7.20 & 6.30 & 3.63 & \\
\hline
\end{tabular}

Table 1. The error of BLE positioning result of participant 1

Since one of the main stations didn't receive the RSSI when participant 2 took the last circle for some trouble, only the former 3 circles would be discussed here. Figure 8 is the visualization of the positioning results of participant 2 at the end of circle 1 and 2 . The improvement is up to $70 \%$, which is demonstrated in Table 2 . The method proposed enhances the accuracy significantly in this experiment.

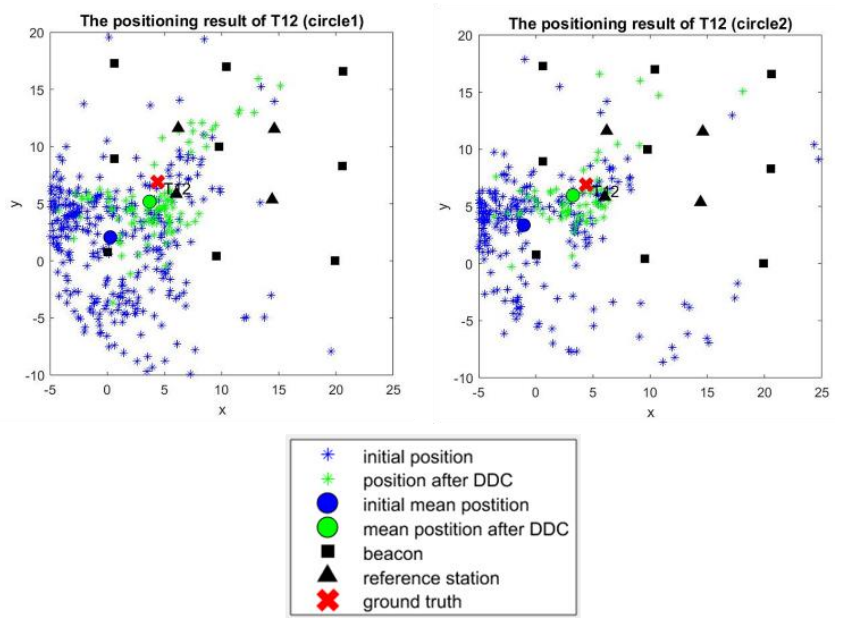

Figure 8. The BLE positioning results of participant 2

\begin{tabular}{|l|l|l|l|l|c|}
\hline \multicolumn{2}{|l|}{$\begin{array}{l}\text { positioning } \\
\text { results }(\mathrm{m})\end{array}$} & RMSE & STD & error & $\begin{array}{c}\text { improvement } \\
(\%)\end{array}$ \\
\hline \multirow{2}{*}{$\begin{array}{l}\text { End of } \\
\text { circle1 }\end{array}$} & original & 9.44 & 6.97 & 6.37 & \multirow{2}{*}{$70.80 \%$} \\
\cline { 2 - 5 } & DDC & 5.78 & 5.50 & 1.86 & \\
\hline \multirow{2}{*}{$\begin{array}{l}\text { End of } \\
\text { cicle2 }\end{array}$} & original & 9.50 & 6.91 & 6.53 & \multirow{2}{*}{$77.18 \%$} \\
\cline { 2 - 5 } & DDC & 4.98 & 4.78 & 1.49 & \\
\hline
\end{tabular}

Table 2. The error of BLE positioning result of participant 2

\subsection{Pedestrian Dead Reckoning}

Figure 9(a) shows the trajectory using the magnetic compass shifting upward with time. The probable cause is the influence of the magnetic field. After updating the coordinate calculated by BLE positioning with DDC in T12, it eliminates the upward shifting. The new trajectory is demonstrated in Figure 9(b). From the Table 3, after the position of the end of circle 1, namely the beginning of circle 2 , is replaced with corresponding BLE results in the previous section, the coordinate of the end of circle 2 will improve $89.92 \%$. The trajectory using gyroscope is depicted in Figure 9(c)(d), but its statistics in Table 4 don't demonstrate the enhancement of accuracy as expected. The reason why the 
accuracy decreases is that the PDR error accumulated with time is still slight and the original results are accurate enough. That is, using the BLE results in the wrong circumstances would worse the PDR results.
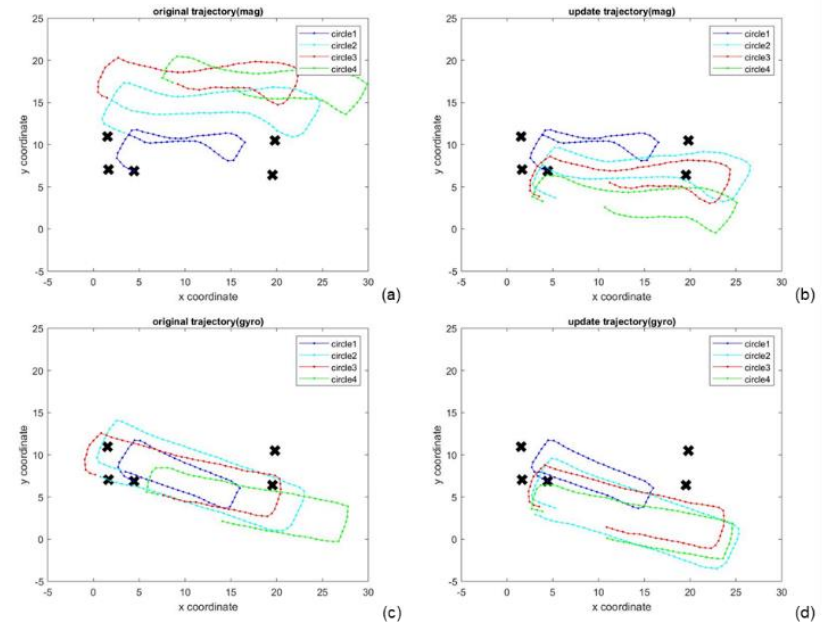

Figure 9. The trajectory of participant 1

\begin{tabular}{|l|l|c|c|}
\hline \multicolumn{2}{|c|}{ PDR using magnetic compass } & Error(m) & $\begin{array}{c}\text { improvement } \\
(\%)\end{array}$ \\
\hline \multirow{2}{*}{$\begin{array}{l}\text { 2nd end } \\
\text { coordinate }\end{array}$} & original & 8.68 & \multirow{2}{*}{$89.92 \%$} \\
\cline { 2 - 3 } & update with BLE & 0.87 & \\
\hline \multirow{2}{*}{$\begin{array}{c}\text { 3rd end } \\
\text { coordinate }\end{array}$} & original & 11.36 & \multirow{2}{*}{$38.72 \%$} \\
\cline { 2 - 3 } & update with BLE & 6.96 & \\
\hline \multirow{2}{*}{$\begin{array}{c}\text { 4th end } \\
\text { coordinate }\end{array}$} & original & 14.73 & \multirow{2}{*}{$48.36 \%$} \\
\cline { 2 - 3 } & update with BLE & 7.61 & \\
\hline
\end{tabular}

Table 3. The error of PDR using magnetic compass of participant 1

\begin{tabular}{|c|l|c|c|}
\hline \multicolumn{2}{|c|}{ PDR using gyroscope } & Error(m) & $\begin{array}{c}\text { improvement } \\
(\%)\end{array}$ \\
\hline \multirow{2}{*}{$\begin{array}{c}\text { 2nd end } \\
\text { coordinate }\end{array}$} & original & 3.68 & \multirow{2}{*}{$-13.57 \%$} \\
\cline { 2 - 3 } & update with BLE & 4.17 & \\
\hline $\begin{array}{c}\text { 3rd end } \\
\text { coordinate }\end{array}$ & original & 3.61 & \multirow{2}{*}{$-134.67 \%$} \\
\cline { 2 - 3 } $\begin{array}{c}\text { 4th end } \\
\text { coordinate }\end{array}$ & update with BLE & 8.48 & \\
\cline { 2 - 3 } & update with BLE & 9.41 & \multirow{2}{*}{$12.77 \%$} \\
\hline
\end{tabular}

Table 4. The error of PDR using gyroscope of participant 1

Figure 10 is the trajectory of participant 2, while Table 5 and Table 6 show the positioning accuracy before and after. In the part of using the magnetic compass, the improvement is about $48 \%$, while in terms of using the gyroscope, the longer the travelled distance is, the more improvement it is. The improvements of circle 2 and 3 are respectively $29 \%$ and $66 \%$, the reason is that the PDR error accumulated with time of circle 3 is larger than that of circle 2. Different from participant 1 , the
BLE results in this experiment are excellent so that the results of PDR greatly improved.
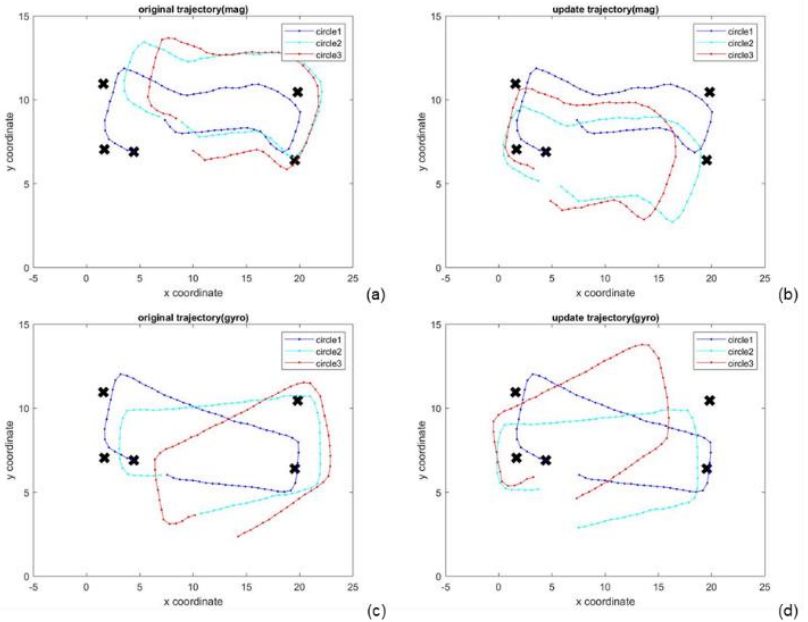

Figure 10. The trajectory of participant 2

\begin{tabular}{|l|l|c|c|}
\hline \multicolumn{2}{|c|}{ PDR using magnetic compass } & Error(m) & $\begin{array}{c}\text { improvement } \\
(\%)\end{array}$ \\
\hline \multirow{2}{*}{$\begin{array}{l}\text { 2nd end } \\
\text { coordinate }\end{array}$} & original & 4.87 & \multirow{2}{*}{$48.18 \%$} \\
\cline { 2 - 3 } & update with BLE & 2.52 & \\
\hline \multirow{2}{*}{$\begin{array}{l}\text { 3rd end } \\
\text { coordinate }\end{array}$} & Original & 5.58 & \multirow{2}{*}{$47.08 \%$} \\
\cline { 2 - 3 } & update with BLE & 2.95 & \\
\hline
\end{tabular}

Table 5. The error of PDR using magnetic compass of participant 2

\begin{tabular}{|l|l|c|c|}
\hline \multicolumn{2}{|l|}{ PDR using gyroscope } & Error(m) & $\begin{array}{c}\text { improvement } \\
(\%)\end{array}$ \\
\hline \multirow{2}{*}{$\begin{array}{l}\text { 2nd end } \\
\text { coordinate }\end{array}$} & original & 7.09 & \multirow{2}{*}{$28.61 \%$} \\
\cline { 2 - 3 } & update with BLE & 5.06 & \multirow{2}{*}{6} \\
\hline \multirow{2}{*}{$\begin{array}{c}\text { 3rd end } \\
\text { coordinate }\end{array}$} & original & 10.81 & \multirow{2}{*}{$65.97 \%$} \\
\cline { 2 - 3 } & update with BLE & 3.68 & \\
\hline
\end{tabular}

Table 6. The error of PDR using gyroscope of participant 2

\section{CONCLUSION}

In terms of indoor positioning with BLE, the proposed method, DDC, enhances the accuracy in the BLE-based indoor positioning system using trilateration technique. As for the PDR, since the environment effect varies the data from the magnetic compass, the enhancement after updating coordinate with BLE can improve the overall accuracy, while the gyroscope is relatively stable in the experimental filed so that the improvement would be noticeable until the error accumulates up to a certain amount, namely the longer distance. The new trajectories are more accurate overall. Nevertheless, setting the threshold of stopping time and travelled distance in the future to know whether to use BLE to update the coordinate is necessary, or the method would worse the results.

\section{ACKNOWLEDGEMENTS}

The authors would acknowledge the supports provided by the Ministry of the Interior. 


\section{REFERENCES}

Basiri, A., Lohan, E. S., Moore, T., Winstanley, A., Peltola, P., Hill, C., Amirian, P., Silva, P., 2017. Indoor location based services challenges, requirements and usability of current solutions. Computer Science Review, 24, 1-12.

Čabarkapa, D., Grujić, I., \& Pavlović, P., 2015. Comparative analysis of the bluetooth low-energy indoor positioning systems. The 12th International Conference on Telecommunication in Modern Satellite, Cable and Broadcasting Services, 76-79.

Chen, R., Pei, L., Chen, Y.,2011. A smart phone based PDR solution for indoor navigation. The 24th International Technical Meeting of the Satellite Division of the Institute of Navigation, 1404-1408.

Kuo, Y. T, Liao, J. K., Chiang, K. W., 2017. Performance Analysis of Indoor Positioning using Differential Distance Correction based on Bluetooth Low Energy. The 10th International Conference on Mobile Mapping Technology, 48-54.

Mautz, R., 2012. Indoor positioning technologies. ETH Zürich.

Murphy, W. S., 2007. Determination of a position using approximate distances and trilateration. Colorado School of Mines.

Velux., 2018. Modern indoor living can be bad for your health: New YouGov survey for VELUX sheds light on risks of the "Indoor Generation". https://www.prnewswire.com/newsreleases/modern-indoor-living-can-be-bad-for-your-health-newyougov-survey-for-velux-sheds-light-on-risks-of-the-indoorgeneration-300648499.html

Zhuang, Y., Yang, J., Li, Y., Qi, L., El-Sheimy, N., 2016. Smartphone-based indoor localization with bluetooth low energy beacons. Sensors, 16(5), 596. 